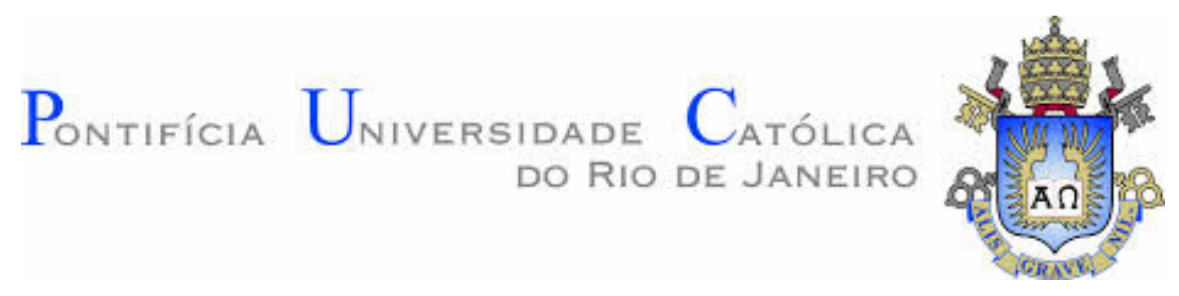

Andres Felipe Cruz Becerra

\title{
Caracterização de PEAD Antes e Após Envelhecimento em Diferentes Meios e Condições
}

Dissertação apresentada como requisito parcial para obtenção do título de Mestre pelo programa de Pósgraduação em Engenharia de Matérias e Processos Químicos e Metalúrgicos da PUC - Rio

Orientador. Prof. José Roberto Moraes d'Almeida 


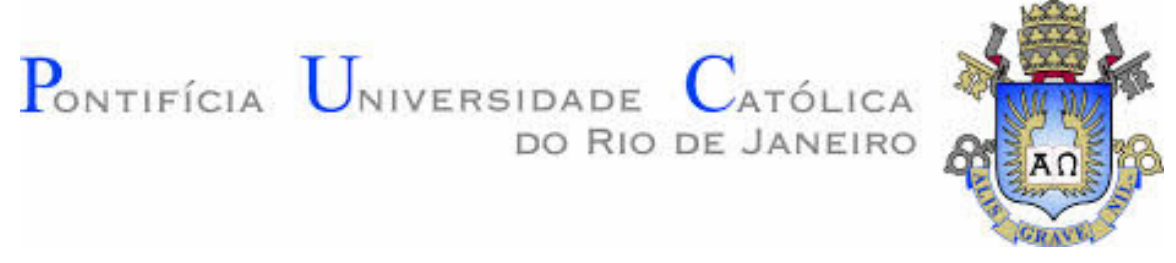

Andres Felipe Cruz Becerra

\title{
Caracterização de PEAD Antes e Após Envelhecimento em Diferentes Meios e Condições
}

\begin{abstract}
Dissertação apresentada como requisito parcial para obtenção do título de Mestre pelo programa de Pósgraduação em Engenharia de Matérias e Processos Químicos e Metalúrgicos da PUC - Rio. Aprovada pela Comissão Examinadora abaixo assinada
\end{abstract}

\begin{abstract}
Prof. José Roberto Moraes d'Almeida Orientador e Presidente Departamento de Engenharia Química de Materiais - PUC - Rio.

Prof. Marcos Henrique de Pinho Maurício Departamento de Engenharia Química de Materiais - PUC - Rio.
\end{abstract}

\section{Prof. - Nara Guidacci Berry Petrobrás-CENPES}

Prof. José Eugenio Leal Coordenador Setorial do Centro Técnico Científico da - PUC - Rio

Rio de Janeiro, 27 Agosto de 2015. 
Todos os direitos reservados. É proibida a reprodução total ou parcial do trabalho sem autorização da universidade, do autor e do orientador.

\section{Andres Felipe Cruz Becerra}

Engenheiro Metalúrgico formado na Universidad Industrial de Santander - Santander, Colômbia 2013.

Ficha Catalográfica

Becerra, Andres Felipe Cruz

Caracterização de PEAD Antes e Após Envelhecimento em Diferentes Meios e Condições / Andres Felipe Cruz Becerra ; orientador: José Roberto Moraes D’Almeida - 2015.

223 f. : il. (color.) ; $30 \mathrm{~cm}$

Dissertação (mestrado)-Pontifícia Universidade Católica do Rio de Janeiro, Departamento de Engenharia Química e de Materiais, 2015.

Inclui bibliografia

1. Engenharia Química - Teses. 2. PEAD. 3. Fluência. 4. Envelhecimento. 5. Comportamento Mecânico. 6. Viscoelasticidade. I. D'Almeida, José Roberto Moraes. II. Pontifícia Universidade Católica do Rio de Janeiro. Departamento de Engenharia Química e de Materiais. III. Título.

CDD: 620.11 
Este trabalho é dedicado a minha esposa Yina Paola Pineda Ariza, minha mãe Luz Amparo, meu pai Samuel Cruz e meus irmãos Edzon e Diana. 


\section{Agradecimentos}

A Deus por sempre estar comigo e me proteger e por cuidar da minha esposa e família em todos os momentos e especialmente durante a minha ausência ficando longe deles.

Ao meu orientador Professor José Roberto Moraes D'Almeida pela orientação, ensinamentos e a grande contribuição para o desenvolvimento deste trabalho, a sua paciência e confiança depositada em mim, durante toda a minha formação como Mestre.

Ao Professor Nicolas Rey do Departamento de Química da PUC - Rio, por facilitar a realização da caracterização espectroscópica FTIR, e ao Jorge pela ajuda e a gentileza na realização da análise.

À professora do laboratório de raios X, Sônia, pela ajuda na realização da caracterização da difração de raios X; e à Margarita e Juliana, pela auxilio no ensaio DSC/TGA.

A minha Esposa Yina Paola Pineda, pelo seu grande amor incondicional, apoio, confiança, paciência e compreensão durante o tempo do mestrado, sentimentos que permitiram me fortalecer e continuar dia a dia.

Aos meus Pais, Samuel Cruz e Luz Amparo Becerra, também e aos meus irmãos, Edzon Geovanny Cruz e Diana Camila Cruz, pelo seu afeto e carinho fornecido.

Aos meus amigos e companheiros de sala: Ronald, Danny, Margarita, Patrícia, Max, Juliana, pela sua ajuda e a sua amizade.

À PUC-Rio, e a coordenação do Departamento de Engenharia Química e de Materiais pela a sua ajuda e orientação e ao Conselho Nacional de Desenvolvimento Científico e Tecnológico (CNPq) pela bolsa outorgada para a realização dos meus estudos de mestrado. 


\section{Resumo}

Becerra, Andres Felipe Cruz; d'Almeida, José Roberto Moraes. Caracterização de PEAD Antes e Após Envelhecimento em Diferentes Meios e Condições. Rio de Janeiro, 2015. 223p. Dissertação de Mestrado Departamento de Engenharia Química e de Materiais, Pontifícia Universidade Católica do Rio de Janeiro.

O interesse deste trabalho está baseado no estudo da influência das diferentes condições e meios de envelhecimento nas propriedades mecânicas, térmicas, estruturais e químicas do polietileno de alta densidade (PEAD) utilizado para a fabricação das tubulações para o transporte de óleo e gás. Foram fabricados corpos de prova com o formato para o teste de tração tipo V segundo a norma ASTM D638. Para o desenvolvimento deste trabalho os corpos de prova foram submetidos a diferentes tipos de envelhecimento em diferentes meios, a saber: i) radiação UV com uma energia radiante de comprimento de onda de $365 \mathrm{~nm}$ e tempos de exposição de 8, 12 e 18 semanas; ii) envelhecimento sob temperatura, os corpos de prova foram imersos em três diferentes tipos de óleo lubrificantes, a 50, 70 e $90{ }^{\circ} \mathrm{C}$ em tempos de 2, 3, 4 e 6 meses; iii) envelhecimento sob pressão, onde os corpos de prova foram submetidos a 7, 14 e 17 bar e imersos em água corrente a $50{ }^{\circ} \mathrm{C}$ durante 1 e 2 semanas e no óleo padrão BASIC OB 440 a $70{ }^{\circ} \mathrm{C}$ durante 1,2 e 5 semanas. As amostras envelhecidas foram caracterizadas por difração de raios $\mathrm{X}$, espectroscopia infravermelha, espectroscopia RAMAN, calorimetria diferencial de varredura, análise termogravimétrica, ensaio de tração, ensaios de fluência, índice de fluidez, microscopia eletrônica de varredura e microscopia óptica. Os resultados das caracterizações das amostras após envelhecimento foram comparados com os resultados obtidos do PEAD virgem, cujas propriedades foram obtidas usando os mesmos métodos de caracterização. Foi avaliado, ainda, o comportamento viscoelástico do PEAD, mediante a simulação matemática dos modelos viscoelásticos, partindo dos resultados experimentais dos testes de fluência antes e após de envelhecimento. Os resultados obtidos demostraram que comportamento mecânico do PEAD após ser envelhecido foi fortemente influenciado pelas diferentes variáveis, como temperatura, pressão, meio e tempo de exposição. 
Sendo que a soma de cada efeito gerado por cada parâmetro contribui para a variação do desempenho mecânico do polímero. Qualquer que seja a variável externa imposta ao PEAD, temperatura, tempo, pressão ou meio de exposição, sempre acarretam alterações nas propriedades mecânicas. Porém, dependendo de qual dos parâmetros mencionados anteriormente seja o predominante, o envelhecimento do PEAD será mais ou menos relevante. Da simulação do comportamento sob fluência, o modelo dos 4 parâmetros foi quem apresentou a melhor descrição gráfica e aproximação matemática do comportamento experimental.

\section{Palavras - Chave}

PEAD; Fluência; Envelhecimento; Comportamento Mecânico; Viscoelasticidade. 


\section{Abstract}

Becerra, Andres Felipe Cruz; d’Almeida, José Roberto Moraes (Advisor). HDPE Characterization before and after aging in different conditions and medium. Rio de Janeiro, 2015. 223p. MSc. Dissertation Departamento de Engenharia Química e de Materiais, Pontifícia Universidade Católica do Rio de Janeiro.

The interest of this work was based on the study of the influence of different aging conditions and medium in mechanical, thermal, structural and chemical properties of high-density polyethylene (HDPE) used for the manufacture of line pipes for transporting oil and gas. Prototypes for type- $\mathrm{V}$ tensile testing according to ASTM D 638. For development this work, the samples were submitted to different types of aging in different medium, namely: i) UV irradiation with a radiant energy with $365 \mathrm{~nm}$ of wavelengths and exposure times of 8, 12 and 18 weeks; ii) For aging under temperature, the samples were immersed in three different types of lubricating oil, to 50, 70 and $90{ }^{\circ} \mathrm{C}$ for periods of 2, 3, 4 and 6 months; iii) aging under pressure, where the samples were submitted to 7,14 and 17 bar and immersed in ordinary water at $50^{\circ} \mathrm{C}$ for 1 and 2 weeks and pattern Oil BASIC B $440-70{ }^{\circ} \mathrm{C}$ for 1,2 and 5 weeks. The aged samples was characterized by X-ray diffraction, infrared spectroscopy, Raman spectroscopy, differential scanning calorimetric, thermal gravimetric analysis, tensile and creep tests, melt flow rate, scanning electron microscopy and optical microscopy. The results of the characterization of the samples after aging were compared with the results obtained from virgin HDPE, whose properties were obtained using the same characterization methods. Besides, was evaluated, the viscoelastic behavior of the HDPE, by mathematical simulation of viscoelastic models, based on experimental results of creep tests before and after aging. The results showed that mechanical behavior of HDPE after aging was strongly influenced by different variables, such as temperature, pressure, medium and exposure time. Since the sum of each effect generated by each parameter contributes to the variation of the mechanical performance of the polymer. Whichever the external variable imposed on HDPE temperature, time, pressure, or exposure medium - always leads to changes in mechanical properties. However, depending on which of the parameters mentioned above is predominant, the aging HDPE will be more or less relevant. 
Simulating the creep behavior, the four parameters model was the one who presented the best graphic and mathematical description approximation of the experimental behavior.

\section{Keywords}

HDPE; Creep; Aging; Mechanical Behavior; Viscoelasticity. 


\section{Sumário}

1.

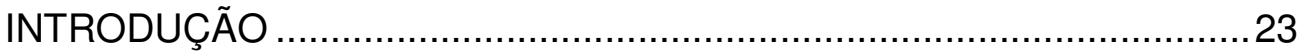

2.

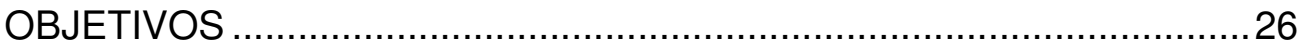

3.

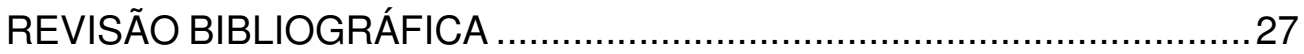

3.1 .

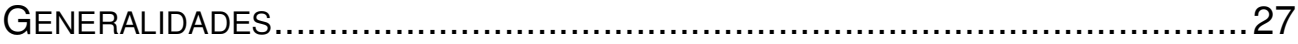

3.1.1._Conceito de Polímero ...............................................27

3.1.2. Copolímeros ........................................................28

3.1.3. Tipos de Polímeros ...............................................29

3.1.4._Cristalinidade dos Polímeros................................... 34

3.2.

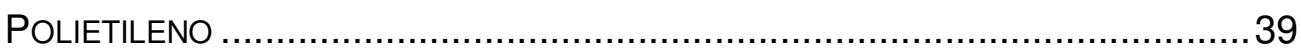

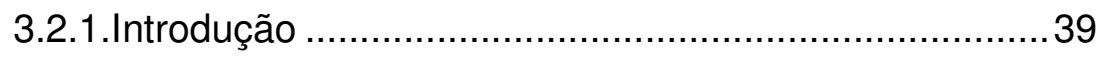

3.2.2._Morfologia Molecular do Polietileno........................... 40

3.2.3 _Estrutura Física do Polietileno .................................... 42

3.2.4_Propriedades do Polietileno .................................... 45

3.2.5_Uso do Polietileno em Tubulações .............................. 46

3.3.

Polietileno de Alta Densidade (PEAD) ...........................................49 49

3.3.1_Classificação do Polietileno de Alta Densidade. ..........50

3.3.2_Propriedades Gerais do Polietileno de Alta Densidade 50

3.3.3_Viscoelasticidade dos Polímeros ............................... 55

3.3.4_Modelos Viscoelásticos .......................................... 62

3.5 .

DEGRADAÇÃO DOS POLÍMEROS............................................................ 72

3.5.1_Fatores Responsáveis da Degradação do PEAD ........73

3.5.2_Tipos de Processos de Degradação .......................... 75

4

MATERIAIS E MÉTODOS EXPERIMENTAIS .................................... 84

4.1.

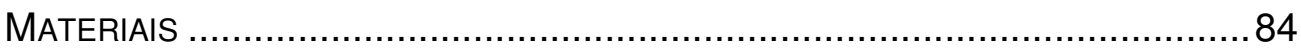

4.2.

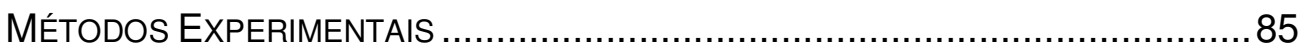

4.2.1.

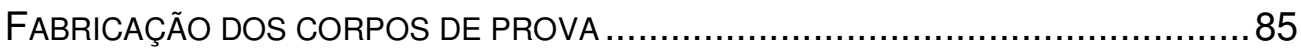

4.2.2_Métodos de Envelhecimento .................................. 87 
4.2.2.1_Envelhecimento em Óleo e Água aquecidos sob

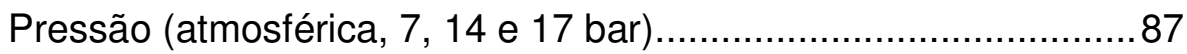

4.2.2.2_Envelhecimento por Radiação Ultravioleta (UV) ........88

4.2.2.3 Envelhecimento em Óleos Aquecidos sobPressão

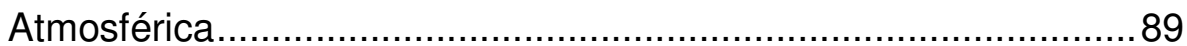

4.3. 90

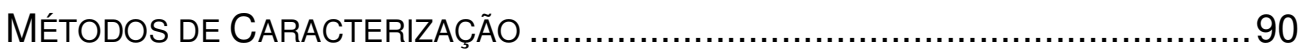

4.3.1. 90

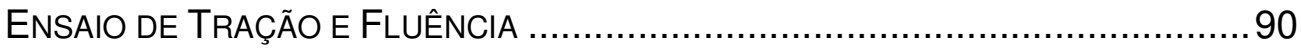

4.3.2.

Difração de Raios X ......................................................... 91

4.3.3._Análise Termogravimétrica (TGA) ...............................93

4.3.4_Calorimetria Diferencial de Varredura (DSC) ...............93

4.3.5._Espectroscopia Infravermelho com Transformada

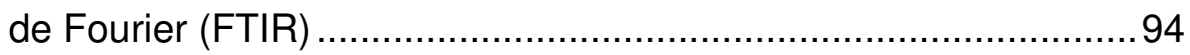

4.3.6_Espectroscopia RAMAN..............................................95

4.3.7Índice de Fluidez (MFI).................................................96

4.3.8_Microscopia Eletrônica de Varredura (MEV - EDX) e

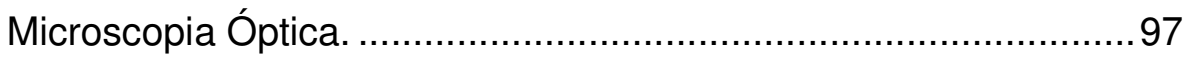

5.

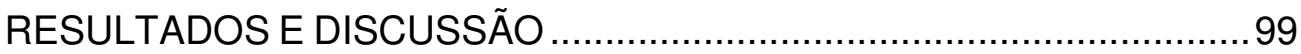

5.1 .

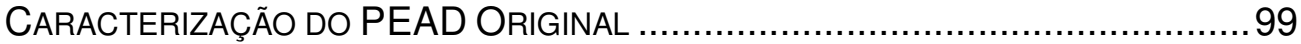

5.1.1._Caracterização Mecânica .............................................99

5.1.2._Caracterização por DRX ......................................... 105

5.1.3._Caracterização Térmica ........................................... 106

5.1.4._Caracterização Química.............................................109

5.2.

CARACTERIZAÇÃo do PEAD ENVELHECIDO EM ÁGUA A $50 \stackrel{\circ}{\circ} \mathrm{C}$, POR 1

SEMANA E SOB PRESSÕES ATMOSFÉRICA, 7 E 14 BAR ................................118

5.2.1._Caracterização Mecânica...........................................118

5.2.2._Caracterização por DRX ........................................ 124

5.2.3._Caracterização Química............................................125

5.3.

CARACTERIZAÇÃo do PEAD ENVELHECIDO EM ÁGUa A 50 드, 14 BAR, DURANTE 1 E 2 SEMANAS...

5.3.1._Caracterização Mecânica..........................................129

5.3.2._Caracterização por DRX ......................................... 136 
5.3.3._Caracterização Química.............................................137

5.3.4._Microscopia Óptica................................................ 138

5.4 .

CARACTERIZAÇÃO do PEAD ENVELHECIDO POR RADIAÇÃO UV ...................139

5.4.1.

CARACTERIZAÇÃO MECÂNICA ......................................................... 139

5.4.1.1.

PROPRIEDAdES À TRAÇÃO................................................................... 139

5.4.1.2.

PROPRIEDADES À FLUÊNCIA .............................................................. 142

5.4.2._Caracterização por DRX ..................................... 149

5.4.3_Caracterização térmica ........................................ 149

5.4.4._Caracterização química........................................ 152

5.5.

CARACTERIZAÇÃO do PEAD ENVELHECIDO EM ÓleO BÁSICO A 70 C, DuRANTE 5 Semanas nas Pressões AtMosfériCA, 7, 14 E 17 BAR. ..........162

5.5.1._Caracterização Mecânica..................................... 162

5.5.2._Caracterização por DRX ....................................173

5.5.3._Caracterização térmica ....................................... 174

5.5.4._Caracterização química........................................177

5.5.4.1._Análise do espectro FTIR e RAMAN ....................177

5.6.

CARACTERIZAÇÃo do PEAD ENVELheCIDO EM Óleos Aquecidos em

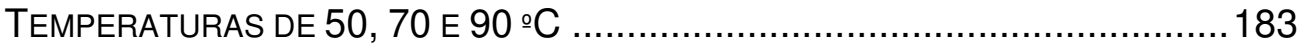

5.6.1.

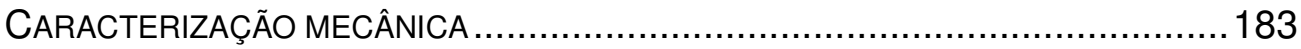

5.6.1.1.

PROPRIEDADES À TRAÇÃO ........................................................ 183

5.6.2_Caracterização por DRX ................................... 195

5.6.3._Caracterização térmica .........................................196

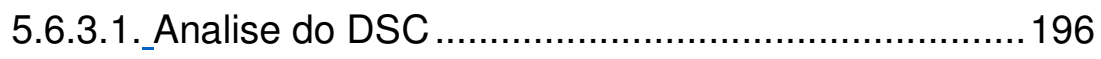

5.6.3.2._Analise do TGA .............................................. 198

5.6.4._Caracterização química........................................200

6.

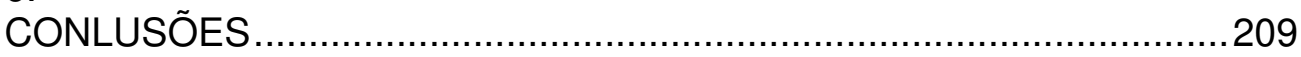

6.1._Sugestões para trabalhos futuros ............................211

7.

REFERENCIAS BIBLIOGRÁFICAS...................................................... 212

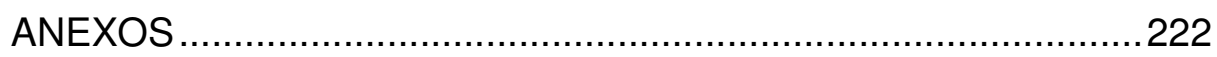




\section{Listado de Figuras}

Figura 1. Diagrama esquemático da obtenção do polímero [8]

Figura 2. Tipo de copolímeros atendendo à disposição relativa de seus monômeros A (Esfera verde) e B (esfera preta); a) copolímero de tipo aleatório, b) copolímero alternado, c) copolímero em bloco e d) copolímero ramificado [7].

Figura 3. Esquemas das diferentes estruturas moleculares dos polímeros: a) polímero linear, b) polímero ramificado, c) polímero com ligações cruzadas, e d) polímero de rede [8].

Figura 4. Esquema de um polímero com estrutura cristalina e estrutura amorfa [8].

Figura 5. Micrografia eletrônica de transmissão de uma estrutura esferulitíca de uma amostra de borracha natural [12].....

Figura 6. Difratograma de raios X do PP esferulitíco [12].

Figura 7. Esquema do termograma característico da técnica de calorimetria exploratória diferencial (DSC) [12].

Figura 8. Variação do módulo de elasticidade $(\mathrm{E})$ em relação à temperatura e um polímero com regiões cristalinas e amorfas [7]...

Figura 9. Representação esquemática da polimerização do polietileno [18] ..... 40

Figura 10. Esquema da estrutura química do polietileno puro [15].

Figura 11. Esquema da estrutura química, com configuração sp3, para os átomos de carbono da cadeia principal de uma molécula de polietileno [15].

Figura 12. Esquema da morfologia de um polímero semicristalino [18].....

Figura 13. Monocristal do polietileno, obtido a partir de uma solução (MEV, 20.000x) [12].

Figura 14. Representação esquemática de uma célula unitária de polietileno em duas vistas a) Ortogonal, b) De topo [4].

Figura 15. Estrutura esquemática de cadeia dobrada de uma lamela de polietileno [20].

Figura 16. Esquema da estrutura esferulitíca presente no polietileno [21].

Figura 17. Tubulações de polietileno de alta densidade (PEAD) utilizadas para o transporte de derivados do petróleo [22]. 
Figura 18. Gráfico para a determinação do valor de MRS, para o PE 80 e PE 100 [9].

Figura 19. Estrutura molecular do PEAD [5].

Figura 20. Fenômenos comuns para materiais viscoelásticos a) elasticidade instantânea, b) Fluência sob tensão constante, c) relaxação de tensão sob deformação constante, d) recuperação elástica e d) Recuperação da deformação, após a retirada a carga [26].

Figura 21. Comportamento de fluência do polietileno [28].

Figura 22. a) Componente elástica (mola) e b) comportamento linear da mola [26].

Figura 23. a) Componente viscosa (amortecedor), b) comportamento linear com tensão constante e c) comportamento do amortecedor com deformação constante [26].

Figura 24. Esquema do Modelo de Maxwell.

Figura 25. a) Aplicação da tensão constante no sistema e b) Comportamento do modelo de Maxwell sob fluência.

Figura 26. Esquema do Modelo de Kelvin - Voight.

Figura 27. a) Aplicação da tensão constante no sistema e b) Comportamento do modelo de Kelvin - Voight sob fluência.

Figura 28. Esquema do modelo dos três parâmetros. 70

Figura 29. Esquema do modelo de quatro parâmetros. 71

Figura 30. Esquema do modelo multi-elementos a) unidades de Kelvin - Voigt conectadas em série e b) unidades do modelo de Maxwell conectadas em paralelo.

Figura 31. Esquema de degradação do polímero.

Figura 32. Ruptura da ligação C-C [39]....

Figura 33. União de substituintes na cadeia principal [39].

Figura 34. Reação sem ruptura da cadeia principal [39].

Figura 35. Representação esquemática da difusão de um liquido dentro de um polímero [35].

Figura 36. Representação esquemática o processo de oxidação dos polímeros. 
Figura 38. Equipamento utilizado na fabricação dos corpos de prova: a)

Extrusora e b) micro injetora empregadas neste trabalho.

Figura 39. Formato esquemático dos corpos de prova, segundo a Norma ASTM D638 [40].

Figura 40. Molde aberto com o corpo de prova injetado.

Figura 41. Autoclave utilizada neste trabalho. 88

Figura 42. Câmara de radiação UV utilizada neste trabalho.

Figura 43. Máquina de ensaio de tração. .90

Figura 44. Difratômetro BRUKER, série D8 Discover, utilizado neste trabalho. .92

Figura 45. Diagrama esquemático da análise dos difratogramas por deconvolução gaussiana...

Figura 46. Espectrômetro infravermelho Perkin Elmer, modelo Spectrum 400...95

Figura 47. Espectrofotômetro Perkin Elmer, modelo Raman Itatian 400. Utilizado neste trabalho.

Figura 48. Plastômetro de extrusão Melt Flow Indexer- Tinius Olsen modelo $\mathrm{MFI}-410$, empregado neste trabalho.

Figura 49. Curva de Tensão (MPa) vs Deformação $(\mathrm{mm} / \mathrm{mm})$ para o PEAD original. 100

Figura 50. Curva do ensaio de fluência para tensões de 3, 6 e $7 \mathrm{MPa}$ no PEAD original.

Figura 51. Esquema do processo de deformação na fluência do PEAD original [8]. 102

Figura 52. Modelagem da fluência do PEAD original para a) $3 \mathrm{MPa}, \mathrm{b}$ ) $6 \mathrm{MPa}$ e c) $7 \mathrm{MPa}$. 104

Figura 53. Difratograma do PEAD original. 106

Figura 54. Deconvolução do difratograma do PEAD original. 106

Figura 55. Curva do DSC do PEAD original. 107

Figura 56. Resultado do ensaio da análise termogravimétrica do PEAD original, a) TGA e b) Derivada do TGA (DTGA). 109

Figura 57. Espectro FTIR e RAMAN do PEAD original.

Figura 58. Representação esquemática das diferentes vibrações das ligações C-H do PEAD [2]. 
Figura 59. Espectro FTIR e RAMAN do Óleo Básico Original.

Figura 60. Deconvolução do espectro FTIR do óleo Básico, nas faixas de a) $650 \mathrm{~cm}^{-1}$ e $1000 \mathrm{~cm}^{-1}$, b) $1250 \mathrm{~cm}^{-1}$ e $1550 \mathrm{~cm}^{-1}$ e c) $2800 \mathrm{~cm}^{-1} \mathrm{e}$ $3050 \mathrm{~cm}^{-1}$

Figura 61. Deconvolução do espectro FTIR do óleo Básico, nas faixas de a) $1160 \mathrm{~cm}^{-1}$ e $1280 \mathrm{~cm}^{-1}$ e b) $1650 \mathrm{~cm}^{-1}$ e $1850 \mathrm{~cm}^{-1}$

Figura 62. Deconvolução do espectro RAMAN do óleo Básico, nas faixas de a) $1160 \mathrm{~cm}^{-1}$ e $1280 \mathrm{~cm}^{-1}$ e b) $1650 \mathrm{~cm}^{-1}$ e $1850 \mathrm{~cm}^{-1}$.

Figura 63. Resultados dos Ensaios de Tração em Função da Pressão (bar) a) Limite de Escoamento (MPa), b) Módulo de Elasticidade (MPa) e c) Deformação Máxima.

Figura 64. Índice de fluidez do PEAD envelhecido a em água, a $50 \stackrel{\circ}{\circ}, 1$ semana e pressões atmosférica, 7 e 14 bar.

Figura 65. Resultados dos ensaios de fluência em função da pressão (bar)

) Taxa de deformação $\left(s^{-1}\right)$ e b) Deformação inicial.

Figura 66. Difractograma de Raios $X$ de PEAD envelhecido na água, a $T$ $=50 \stackrel{\circ}{\mathrm{C}}, \mathrm{t}=1$ Semana, e pressões atmosférica, 7 e 14 bar.

Figura 67. Espectros FTIR (acima) - RAMAN (abaixo) para o PEAD original e envelhecido na água, $50^{\circ} \mathrm{C}$, durante 1 semana, para pressões atmosférica, 7 bar, 14 bar.

Figura 68. Representação esquemática da formação do álcool. a) geração do radical livre pelo ataque nucleofílico e b) Formação do álcool partir do radical livre [62].

Figura 69. Esquema das reações de degradação do PEAD: a) formação do radical alquila e b) formação do peróxido [62].

Figura 70. a) PEAD original; b) PEAD envelhecido em água, a $50^{\circ} \mathrm{C}$, durante 1 semana; $P=$ atm; $c$ Idem; $P=7$ bar; $d$ Idem; $P=14$ bar.

Figura 71. Ensaios de tração em função do tempo de envelhecimento em água a $50^{\circ} \mathrm{C}$ e 14 bar: a) Limite de escoamento (MPa), b) Módulo de Elasticidade (MPa) e c) Deformação Máxima.

Figura 72. Índice de fluidez do PEAD envelhecido a em água, a $50 \stackrel{\circ}{\circ}, P=14$ bar e tempo de imersão 1 e 2 semanas.

Figura 73. Resultados dos ensaios de fluência em função do tempo de envelhecimento (semanas) a) Taxa de deformação $\left(s^{-1}\right)$ e b) Deformação inicial.

Figura 74. Difractograma de Raios $X$ de PEAD envelhecido na água, a $T$ $=50^{\circ} \mathrm{C}, \mathrm{P}=14$ Bar e tempos de 1 e 2 semanas. 
Figura 75. Espectros FTIR (acima) - RAMAN (abaixo) para o PEAD original

e envelhecido na agua, $50^{\circ} \mathrm{C}, 14$ Bar, durante 1 e 2 semanas.

Figura 76. a) e b) Fotografias do PEAD após de ser envelhecido em uma $T=50^{\circ} \mathrm{C}, \mathrm{t}=2$ Semanas e $\mathrm{P}=14$ bar.

Figura 77. Ensaio de tração em função do tempo de exposição à radiação UV,

a) Limite de Escoamento (MPa), b) Módulo de Elasticidade (MPa) e c)

Deformação Máxima.

Figura 78. Índice de fluidez do PEAD envelhecido na radiação UV, durante 8, 12

e 18 semanas.

Figura 79. Taxa de deformação $\left(\mathrm{s}^{-1}\right)$ em função do tempo de exposição à radiação UV para a) $3 \mathrm{MPa}$ b) $6 \mathrm{MPa}$ e c) $7 \mathrm{MPa}$..

Figura 80. Deformação inicial em função do tempo de exposição à radiação UV para a) $3 \mathrm{MPa}$ b) $6 \mathrm{MPa}$ e c) $7 \mathrm{MPa}$.....

Figura 81. Taxa de deformação $\left(\mathrm{s}^{-1}\right)$ em função da tensão aplicada, a) $3 \mathrm{MPa}$ b) $6 \mathrm{MPa}$ e c) $7 \mathrm{MPa}$.

Figura 82. Deformação inicial em função da tensão aplicada, a) 3 MPa b) $6 \mathrm{MPa}$ e c) $7 \mathrm{MPa}$.

Figura 83. Difratograma de Raios X de PEAD envelhecido na radiação UV. ...149

Figura 84. Resultado do ensaio DSC para o PEAD envelhecido na radiação

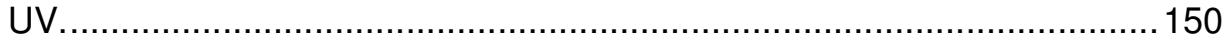

Figura 85. Resultado do ensaio TGA para o PEAD envelhecido na radiação UV

Figura 86. Resultado da derivada do TGA do PEAD envelhecido na radiação UV.

Figura 87. Deconvolução do espectro RAMAN do PEAD exposto a 18 semanas na radiação UV, na banda de $1000 \mathrm{~cm}^{-1}$ e $1600 \mathrm{~cm}^{-1}$

Figura 88. Representação esquemática da reação tipo Norrish II [73].

Figura 89. Representação esquemática da reação de ecsição tipo Beta [73]. . 154

Figura 90. Espectro FTIR e RAMAN do PEAD envelhecido na radiação UV, analisado entre um comprimento de onda de $680 \mathrm{~cm}^{-1}$ e $950 \mathrm{~cm}^{-1}$.

Figura 91. Espectro FTIR e RAMAN do PEAD envelhecido na radiação UV, analisado entre um comprimento de onda de $1250 \mathrm{~cm}^{-1} \mathrm{e} 1750 \mathrm{~cm}^{-1}$.

Figura 92. Representação esquemática da reação Norrish I [76]. 
Figura 93. Representação esquemática fotólise do hidroperóxido seguido da dismutação [75].

Figura 94. Representação esquemática da terminação tipo RUSSEL [75]....... 158

Figura 95. Espectro FTIR e RAMAN do PEAD envelhecido na radiação UV, analisado entre um comprimento de onda de $2700 \mathrm{~cm}^{-1}$ e $3400 \mathrm{~cm}^{-1}$

Figura 96. Micrografias das amostras do PEAD original (a, b, c) e envelhecido na radiação UV durante 8 semanas $(d, e, f), 12$ semanas $(g, h, i)$ e 18 semanas (j, $\mathrm{k}, \mathrm{l})$ a diferentes aumentos 500X, 4000X e 10000X respetivamente.

Figura 97. Resumo dos ensaios de tração, a) Limite de Escoamento (MPa), b) Módulo de Elasticidade (MPa) e c) Deformação Máxima. 163

Figura 98. Curva da porcentagem do peso do PEAD envelhecido sob diferentes pressões.

Figura 99. Fotografias do a) PEAD original e das amostras do PEAD extraídas após do envelhecimento no óleo Básico aquecido a $70 \stackrel{\circ}{ } \mathrm{C}$ e pressões: b) atmosférica, c) 7 bar, d) 14 bar, e) 17 bar.

Figura 100. Índice de fluidez do PEAD envelhecido sob pressão em contato com o óleo básico.

Figura 101. Taxa de deformação $\left(\mathrm{s}^{-1}\right)$ em função da pressão a) a $\left.3 \mathrm{MPa}, \mathrm{b}\right)$ $6 \mathrm{MPa}$ e c) $7 \mathrm{MPa}$.

Figura 102. Deformação Inicial em função da pressão (bar) a) $3 \mathrm{MPa}$, b) $6 \mathrm{MPa}$ e c) $7 \mathrm{MPa}$.

Figura 103. Taxa de deformação $\left(\mathrm{s}^{-1}\right)$ em função da tensão aplicada (MPa)

a) $\mathrm{P}$ atmosférica, b) 7 bar e c) 14 bar e d) 17 bar.

Figura 104. Deformação inicial em função da Tensão aplicada (MPa) a) P atmosférica, b) 7 bar, c) a 14 bar e d) 17 bar.

Figura 105. Difratograma d PEAD envelhecido no óleo, a $\mathrm{T}=70 \stackrel{\circ}{ } \mathrm{C}$, durante 5 semanas, e pressões atmosférica, 7, 14 e 17 bar.

Figura 106. Curva do DSC do PEAD envelhecido sob pressão.

Figura 107. Curva do TGA do PEAD envelhecido sob pressão.

Figura 108. Curva da derivada do TGA (DTGA) do PEAD envelhecido sob pressão.

Figura 109. Deconvolução do espectro RAMAN do PEAD exposto à pressão de $17 \mathrm{bar}$, na banda de $1000 \mathrm{~cm}^{-1}$ e $1600 \mathrm{~cm}^{-1}$.

Figura 110. Espectro FTIR e RAMAN do PEAD envelhecido sob pressão, analisado para a banda dentre 680 e $950\left(\mathrm{~cm}^{-1}\right)$. 
Figura 111. Espectro FTIR e RAMAN do PEAD envelhecido sob pressão, analisado para a banda dentre 950 e $1600\left(\mathrm{~cm}^{-1}\right)$.

Figura 112. Espectro FTIR e RAMAN do PEAD envelhecido sob pressão, analisado para a banda dentre $2700 \mathrm{~cm}^{-1}$ e $3700 \mathrm{~cm}^{-1}$.

Figura 113. Limite de escoamento do PEAD envelhecido a $50 \stackrel{\circ}{\mathrm{C}}, 70 \stackrel{\circ}{\circ} \mathrm{e}$ $90{ }^{\circ} \mathrm{C}$, a) óleo Básico, b) óleo Exterram e c) óleo Lubrax.

Figura 114. Modulo de Young do PEAD envelhecido a $50^{\circ} \mathrm{C}, 70^{\circ} \mathrm{C}$ e $90^{\circ} \mathrm{C}$,

a) óleo Básico, b) óleo Exterram e c) óleo Lubrax.

Figura 115. Deformação máxima do PEAD envelhecido a $50 \stackrel{\circ}{\circ}, 70{ }^{\circ} \mathrm{C}$ e $90 \stackrel{\circ}{ } \mathrm{C}$, a) óleo Básico, b) óleo Exterram e c) óleo Lubrax.

Figura 116. Superfícies das amostras do PEAD envelhecido por 6 meses, em Óleo Exterram a) $70 \stackrel{\circ}{\circ}$, b) $90^{\circ} \mathrm{C}$ e Óleo Lubrax c) $70^{\circ} \mathrm{C}$, d) $90^{\circ} \mathrm{C} \ldots . .187$

Figura 117. Esquema da reação de solvolise dos polímeros [38].

Figura 118. Mudança da cor do PEAD com o aumento da temperatura para 6 meses de envelhecimento.

Figura 119. Índice de fluidez do PEAD envelhecido a $50^{\circ} \mathrm{C}, 70^{\circ} \mathrm{C}$ e $90^{\circ} \mathrm{C}$, a) óleo Básico, b) óleo Exterram e c) óleo Lubrax.

Figura 120. Taxa de deformação do PEAD envelhecido a $50^{\circ} \mathrm{C}, 70^{\circ} \mathrm{C}$ e $90{ }^{\circ} \mathrm{C}$, a) óleo Básico, b) óleo Exterram e c) óleo Lubrax.

Figura 121. Deformação inicial do PEAD envelhecido a $50 \stackrel{\circ}{ } \mathrm{C}, 70{ }^{\circ} \mathrm{C}$ e $90 \stackrel{\circ}{\circ}$,

a) óleo Básico, b) óleo Exterram e c) óleo Lubrax.

Figura 122. Difratograma PEAD envelhecido na temperatura no óleo básico durante 6 meses.

Figura 123. Resultado do ensaio DSC para o PEAD envelhecido no óleo Básico.

Figura 124. Resultado do ensaio TGA para o PEAD envelhecido no óleo Básico.

Figura 125. Resultado do ensaio DTGA para o PEAD envelhecido no óleo Básico.

Figura 126. Espectro FTIR e RAMAN do PEAD envelhecido durante 6 meses, no óleo Exterram, para as temperaturas de $50^{\circ} \mathrm{C}, 60^{\circ} \mathrm{C}$ e $70{ }^{\circ} \mathrm{C}$.

Figura 127. Deconvolução da banda do espectro RAMAN para o PEAD envelhecido durante 6 meses, no óleo Exterram, na temperatura de $90 \stackrel{\circ}{\circ}$, a) $200 \mathrm{~cm}^{-1}-1100 \mathrm{~cm}^{-1}$, b) $2200 \mathrm{~cm}^{-1}-2600 \mathrm{~cm}^{-1}$ 202

Figura 128. Esquema de estrutura do Tiocetais [78]. 203 
Figura 129. Esquema do composto organo-fosforado [78]

Figura 130. Mudança na coloração dos óleos utilizados, com o aumento de temperatura no tempo de envelhecimento.

Figura 131. Espectro FTIR e RAMAN do óleo Básico após de ser envelhecido

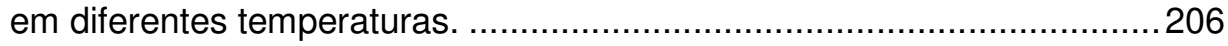

Figura 132. Espectro FTIR e RAMAN do óleo Lubrax após de ser envelhecido em diferentes temperaturas. ............................................................20

Figura 133. Espectro RAMAN do óleo Exterram a $50 \stackrel{\circ}{\circ}$, na faixa entre

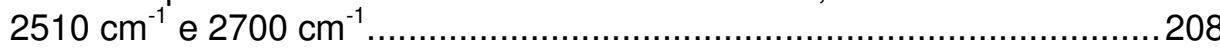




\section{Listado de Tabelas}

Tabela 1. Variação das propriedades do polietileno em função do grau de cristalinidade [15].

Tabela 2. Principias propriedades dos diferentes tipos de polietileno [15].

Tabela 3. Efeito das ramificações sobre as propriedades mecânicas do polietileno [23].

Tabela 4. Propriedades do PEAD empregado neste trabalho fornecidos pela empresa BRASKEM.

Tabela 5. Propriedades mecânicas do PEAD original. 100

Tabela 6. Valores comparativos do ensaio de fluência do PEAD original. Ajuste dos dados experimentais com o modelo de 4 parâmetros.

Tabela 7. Valores dos parâmetros obtidos dos modelos viscoelásticos aplicados à modelagem do comportamento à fluência do PEAD original. 105

Tabela 8. Grau de cristalinidade do PEAD envelhecido na água, a $\mathrm{T}=50 \stackrel{\circ}{\circ}$ , $\mathrm{t}=1$ Semana e pressões atmosférica, 7 e 14 bar, calculado pelo método DRX.

Tabela 9. Componentes do modêlo de 4 parâmetros do PEAD original e envelhecido na água a $50^{\circ} \mathrm{C}, 1$ Semana e a pressões atmosférica, e 14 Bar. Tensão aplicada de $6 \mathrm{MPa}$.

Tabela 10. Grau de cristalinidade do PEAD envelhecido na água, a $\mathrm{T}=50{ }^{\circ} \mathrm{C}$ , $\mathrm{P}=14$ bar, e tempos de imersão de 1 e 2 semanas.

Tabela 11. Componentes do modelo de 4 parâmetros, obtidos a partir da modelagem do PEAD original e envelhecido na água a 50ํㄷ, $14 \mathrm{Bar}$, durante 1 e 2 semanas.

Tabela 12. Grau de cristalinidade do PEAD envelhecido na radiação UV durante 8,12 e 18 semanas.

Tabela 13. Componentes do modelo de 4 parâmetros, do PEAD original e envelhecido naradiação UV, para $3 \mathrm{MPa}$.

Tabela 14. Componentes do modelo de 4 parâmetros, do PEAD original e envelhecido naradiação UV, para $6 \mathrm{MPa}$. 
Tabela 15. Componentes do modelo de 4 parâmetros, do PEAD original e envelhecido naradiação UV, para $7 \mathrm{MPa}$.

Tabela 16. Dados de interesse extraídos do ensaio DSC do PEAD envelhecido na radiação UV.

Tabela 17. Grau de cristalinidade do PEAD envelhecido no óleo, a $T=70 \stackrel{\circ}{C}$ , 5 semanas, e pressões atmosférica, 7,14 e 17 bar, calculado pelo método DRX.

Tabela 18. Componentes do modelo de 4 parâmetros do PEAD original e envelhecido sob pressão, para $3 \mathrm{MPa}$.

Tabela 19. Componentes do modelo de 4 parâmetros do PEAD original e envelhecido sob pressão, para $6 \mathrm{MPa}$.

Tabela 20. Componentes do modelo de 4 parâmetros do PEAD original e envelhecido sob pressão, para $7 \mathrm{MPa}$.

Tabela 21. Dados de interesse extraídos ensaio DSC do PEAD envelhecido sob pressão.

Tabela 22. Grau de cristalinidade do PEAD envelhecido na temperatura durante 6 meses, nos diferentes óleos.

Tabela 23. Componentes do modelo de 4 parâmetros, do PEAD original e envelhecido no óleo básico em função da temperatura, para 6 meses e $3 \mathrm{MPa}$.

Tabela 24. Componentes do modelo de 4 parâmetros, do PEAD original e envelhecido no óleo básico em função da temperatura, para 6 meses e $6 \mathrm{MPa}$.

Tabela 25. Componentes do modelo de 4 parâmetros, do PEAD original e envelhecido no óleo básico em função da temperatura, para 6 meses e $7 \mathrm{MPa}$.

Tabela 26. Dados de interesse extraídos ensaio DSC do PEAD envelhecido no óleo Básico.

Tabela 27. Temperatura da máxima velocidade de perda de massa do PEAD envelhecido na temperatura. 